\title{
Combination of High Dose Hypofractionated Radiotherapy with Anti-PD1 Single Dose Immunotherapy Leads to a Th1 Immune Activation Resulting in a Complete Clinical Response in a Melanoma Patient
}

\author{
Clara Milhem ${ }^{1,2,+}$, Olivier Moralès $1,3,+\left(\mathbb{C}\right.$, Céline Ingelaere ${ }^{2}$, David Pasquier ${ }^{4}$, \\ Serge Mordon ${ }^{1}\left(\mathbb{D}\right.$, Laurent Mortier ${ }^{5}$, Xavier Mirabel ${ }^{4, *,+}$ and Nadira Delhem ${ }^{1, *, \dagger}$ \\ 1 INSERM, CHU-Lille, U1189-ONCO-THAI-Assisted Laser Therapy and Immunotherapy for Oncology, \\ University of Lille, F-59000 Lille, France; clara.milhem@immune-insight.com (C.M.); \\ olivier.morales@ibl.cnrs.fr (O.M.); serge.mordon@inserm.fr (S.M.) \\ 2 Immune Insight, Institut de Biologie de Lille, 59021 Lille, France; ingelaereceline@gmail.com \\ 3 CNRS UMS 3702, Institut de Biologie de Lille, 59021 Lille, France \\ 4 Service de Radiothérapie, Centre Oscar Lambret, 59000 Lille, France; d-pasquier@o-lambret.fr \\ 5 Service de Dermatologie, Hôpital Huriez, 59000 Lille, France; Laurent.MORTIER@CHRU-LILLE.FR \\ * Correspondence: X-Mirabel@o-lambret.fr (X.M.); nadira.delhem@ibl.cnrs.fr (N.D.); \\ Tel.: +33-3-20-29-55-26 (X.M.); +33-3-20-87-12-53 (N.D.); Fax: +33-3-20-87-10-19 (N.D.) \\ + These authors have equally contributed.
}

Received: 15 July 2020; Accepted: 8 September 2020; Published: 15 September 2020

check for updates

\begin{abstract}
The development of immunotherapy has recently modified the anti-tumor therapeutic arsenal; particularly, immune checkpoint inhibitors have led to a significant increase in overall survival. The current challenge is now to select good responder patients by identifying early biomarkers to propose therapeutic combinations that potentiate the efficacy of the therapy. Here we report the case of a 60-year-old man with superficial melanoma treated with high-dose hypo fractionated radiotherapy (H-SRT) combined with a single dose of anti-PD1 immunotherapy (Nivolumab) for a metastatic lymph node recurrence due to cancer progression. In this study, we present the results obtained regarding the activation of the Th1 immune response after H-SRT treatment followed by anti PD-1 therapeutic protocol. These results were correlated with clinical data to identify potential immunological biomarkers of treatment efficacy. This exceptional case report shows that a combination of H-SRT with a single dose of anti-PD1 immunotherapy may allow a better activation of the immune response in favor of a complete clinical response.
\end{abstract}

Keywords: stereotaxic radiotherapy; immunotherapy; anti-PD1; melanoma; immune response

\section{Introduction}

Melanoma is the most common lethal skin cancer form. For malignant melanoma, most cases are diagnosed at an early stage, when surgical excision can be curative. However, some patients can already be at a metastatic stage at diagnosis, and some develop metastases after their initial treatment. Radiation therapy is one of the therapeutic options that can be used to treat it or as an adjuvant way to combat it [1].

Cancer cells are no longer exclusively characterized by genomic alterations' acquisition, but also by their ability to escape immune surveillance [2]. Indeed, the anti-tumor immune response develops in three successive phases, the so called 3E theory redefined by Dunn et al. The Elimination phase is the 
first one during which immune cells recognize and manage tumor cells' elimination. The second one is the Equilibrium phase during which the persistent tumor cells are dormant or continue to accumulate genetic abnormalities. Furthermore, finally, the Escape phase when the tumor overpasses the immune system, proliferates and spreads [3,4].

Recently radiotherapy protocols combining high dosed sessions over a shorter total treatment duration have emerged and are called high dose hypofractionated radiotherapy (H-SRT). Stereotactic irradiation techniques allow better targeting of the tumor, thus making it possible to administer higher doses of ionizing radiation [5]. Preclinical data suggest that high doses per fraction generate a superior immunological response, responsible for an abscopal effect [6]. Described for the first time in the 1950s, the abscopal effect is characterized by tumor regression at an irradiated distance site [7]. The mechanisms of this process are still unclear, but greater antigens and danger signals release (as Calreticulin, damage-associated molecular pattern molecules, high-mobility group box 1 and adenosine triphosphate) would allow the activation of the immune system [8].

Metastatic melanoma treatment has recently been improved by targeted therapies and immunotherapy progress. Most tumor cells express antigens recognizable by the immune system. Nevertheless, some mechanisms permit tumors to escape immunosurveillance such as regulatory $\mathrm{T}$ cells and immune check point (ICP) activation.

ICP (CTLA-4, PD-1, PD-L1 among others) can be blocked by immune checkpoint inhibitors (ICI). By blocking the brakes, immune system reactivation becomes possible and tumor cell effective targeting is allowed. Such strategies rely on specific monoclonal antibodies $(\mathrm{mAb})$ used to specifically target and inhibit ICP. Anti-PD-1 and the anti-PD-1 ligand (PD-L1) are the most used $\mathrm{mAb}$ in melanoma. In fact, unlike normal cells, cancer cells express more often PD-L1 [9] and PD-1 is also expressed on Tregs in association with tumor escape $[10,11]$. Therefore, blocking these ICI would restore a good immune response, that will be fundamental to cancer treatment.

There are an increasing number of studies indicating that combining radiotherapy with immunotherapy would stimulate the abscopal effect [12]. Indeed, radiation therapy is a particularly promising candidate for association with the ICI [13]. In fact, RT effectiveness is well established; it is an accessible and relatively economical procedure with limited and manageable side effects. It is now clear that RT has multi-level immunomodulatory effects. Those effects, which emerge locally in the irradiated field, also have a systemic impact and can be exploited to boost the therapeutic activity of systemic treatments. Some of these effects have been identified as able to increase antigen presentation, NK cell activation and IFN release. H-SRT seems to have a higher immunostimulatory effect than standard RT, particularly in inducing a Th1 response [13]. Clinical trials are underway to validate this association [14], but there is no integrated analysis of underlying cellular, molecular and systemic mechanisms.

Other recent advances confirm the interest of a synergy between radiotherapy and immunotherapy via the abscopal effect $[15,16]$. One of the first studies of anti-PD-1 (Nivolumab@) and radiotherapy combination in patients with metastatic melanoma showed a $46 \%$ response rate on non-irradiated lesions. Irradiated lesion response rate was $44 \%$ for those who received sequential RT and then PD-1 treatment, and $64 \%$ for those who received concomitant treatment [17]. However, the extent to which radiation therapy is involved in these distant responses is not yet known.

In this context, this case report study aims to evaluate the regulation of the immune response upon H-SRT followed by anti PD-1 therapeutic protocol and to correlate immunological and biological data with clinical outcomes in order to identify potential biomarkers of Th1 immune activation and treatment efficacy. 


\section{Case Presentation}

\subsection{Patient's Outcome after High Dose Hypofractionated Radiotherapy}

The patient is a 60 year-old-man, monitored since 2003 for a superficial spreading melanoma on the right leg, firstly treated by surgical excision and lymph node dissection.

The lesion was not BRAF mutated and was classified as high risk with a level IV of Clark, lymph node involvement and with a Breslow index of $1.8-\mathrm{mm}$. Histological analysis did not show ulceration. Right inguinal sentinel lymph node analysis highlighted micrometastases on the three removed nodes $(3 \mathrm{~N}+\mathrm{CR}-/ 3 \mathrm{~N})$. The patient undergoes lymph node dissection $(5 \mathrm{~N}-/ 5 \mathrm{~N})$. He received Interferon- $\alpha$ adjuvant therapy for 18 months.

He presented two skin recurrences in 2011 and 2015 each treated by surgical excision. In 2016, he presented a third recurrence with popliteal lymphadenopathy (Figure 1A) with radiotherapy indication. He was then included in the EarlyBio clinical trial (ClinicalTrials.gov Identifier: NCT02439008) in March 2016.

A.

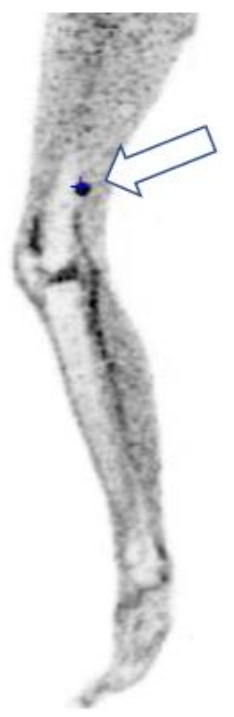

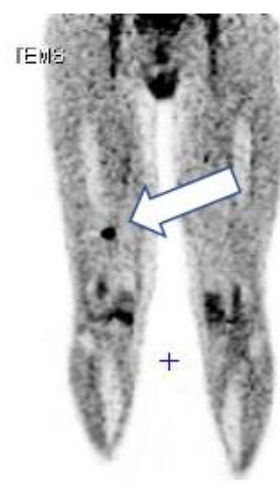

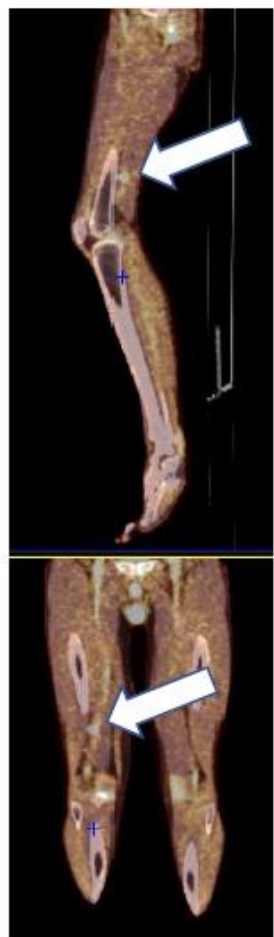

B.

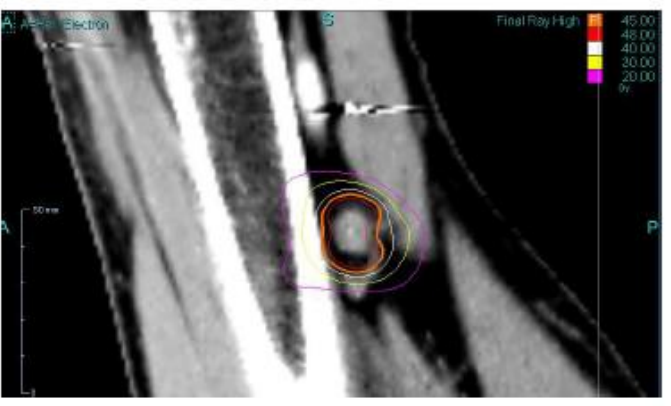

C.

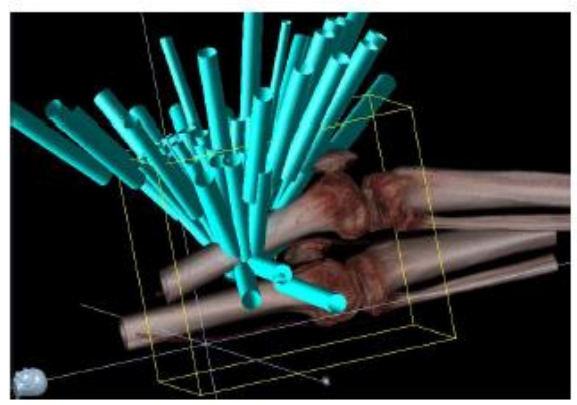

Figure 1. Patient's right leg positron emission tomography (TEP) image (17/02/2016) (A), image of isodose curve around the lesion (B) and visualization of the 65 irradiation ray $(\mathbf{C})$ White arrow point patients irradiated tumor.

The patient's lesion was targeted with high dose hypo fractionated radiotherapy (15 grays), three times during one-week (March 11th, 13th and 17th) using the Cyberknife (Accuray, Sunnyvale, 
CA, USA) robot in the Centre Oscar Lambret anti-cancer center (Lille). Figure 1B shows isodose curves surrounding the lesion and Figure $1 \mathrm{C}$ shows the 65-irradiation rays. Both figures highlight a high radiation impact on the lesion as well as an excellent healthy tissues protection. Days following irradiation the patient presented chills and vitiligo, symptomatology that he had already presented during interferon alpha treatment.

Clinical and imaging evaluations were performed 3 months after the end of radiotherapy, the patient presented a dissociated response with regression of the irradiated lesion but a progression of a subcutaneous nodule, not visible at the imagery but palpable under the skin.

\subsection{Complete Clinical Response after Combination of High Dose Hypofractionated Radiotherapy with Anti-PD1 Single Dose Immunotherapy}

A treatment change was decided, and he received a first injection of Nivolumab (OPDIVO ${ }^{\circledR}$ Bristol-Myers Squibb) at a dose of $3 \mathrm{mg} / \mathrm{kg}$, administered as a 60-min intravenous infusion, shortly after (June 2016).

Sub-cutaneous metastasis excision was programmed in July 2016, but, unexpectedly, the patient presented a complete response after this single injection; the surgery was then cancelled. The treatment was not renewed due to poor tolerance (acute polyarthritis stage 3 development). Clinical and imaging evaluations showed a complete response at 9 and 12 months after treatment with strong decrease of fixation on the irradiated lymphadenopathy and disappearance of right thigh suspicious subcutaneous fixation. He did not receive any new treatment since then. The patient's last TEP (March 2020) did not show any abnormal fixation meaning that total response is still maintained 4 years after the last treatment.

\subsection{Peripheral Immune Cell Activation after Combination of High Dose Hypofractionated Radiotherapy with} Anti-PD1 Single Dose Immunotherapy

Blood samples were collected on dry and EDTA tubes according following the following timeline: before treatment (T0), 15 min after H-SRT session (T1, T2, T3), one week (T4), 3, 6, 9 months (T4, T5, T6, T7) and one year (T8) after the last sequence of H-SRT.

Peripheral blood mononuclear cells (PBMCs) were isolated by density gradient from fresh blood collected in two EDTA tubes (Greiner Bio One Vacuette ${ }^{\circledR}$, Les Ulis, France) using lymphocyte separation medium (Eurobio, Les Ulis, France). The PBMC ring was recovered after centrifugation and PBMCs were washed two times in PBS (GIBCO, Life Technologies, Paisley, UK).

After counting, at least 4 million of PBMCs were studied by flow cytometry using FACS Canto II flow cytometer powered by FacsDiva software (BD Biosciences, Franklin Lakes, NJ, USA). PBMCs were split in 4 panels to be stained with fluorochrome-conjugated $\mathrm{mAbs}$ or corresponding isotype control mAbs as describe in Table 1 (Miltenyi Biotec, Bergisch Gladbach, Germany). Data analysis was made using FlowJo software (ACEA Biosciences, San Diego, CA, USA). Results were presented as population frequency or as median fluorescence intensity (MFI) using Prism 8 (Graphpad Software Inc, San Diego, CA, USA).

Overall, after the first H-SRT session (T1), a decrease is observed in different immune cell sub-populations compared to T0. However, their frequencies increase at T2 and remain stable until T4 (Figure 2).

After the Nivolumab introduction (T6), most of the immune sub-populations continue to increase. This is more important for CD4+, CD14+ and CD19+ (Figure 2A,D,E). This increase is found at T7, with a higher proportion for $\mathrm{CD} 11 \mathrm{c}+$ dendritic cells. At the end of the study all populations decrease with a return to proportions comparable to those observed at T0, except for a larger CD14+ monocyte population (Figure 2). 
Table 1. Flow cytometry panel with antibodies and corresponding isotypes control.

\begin{tabular}{ccc}
\hline Panel & Antibodies & Isotypes \\
\hline \multirow{5}{*}{ Cell type } & CD4 (VIT4)-VioBlue & Mouse IgG2a-VioBlue \\
CD8-VioGreen & Mouse IgG2a-VioGreen \\
CD19-VioBright FITC & Mouse IgG1-VioBright FITC \\
& CD14-PE & Mouse IgG2a-PE \\
CD3-PE-Vio770 & Mouse IgG2a-PE-Vio770 \\
& CD335 (NKp46)-APC & Mouse IgG1-APC \\
CD11c-APC-Vio770 & Mouse IgG2b-APC-Vio770 \\
CD4 (VIT4)-VioBlue & Mouse IgG2a-VioBlue \\
& CD30-APC-Vio770 & Mouse IgG1-APC-Vio770 \\
CD69-PE-Vio770 & Mouse IgG1-PE-Vio770 \\
& Anti-HLA-DR-PerCP-Vio700 & Mouse IgG2a-PerCP-Vio770 \\
CD152-APC & Mouse IgG2a-APC \\
CDctivation & REA Control (S)-PE \\
& CD25-VioBright FITC & Mouse IgG2b-VioBright FITC \\
& CD8-VioGreen & Mouse IgG2a-VioGreen \\
& CD4 (VIT4)-VioBlue & Mouse IgG2a-VioBlue \\
& CD25-VioBright FITC & Mouse IgG2b-VioBright FITC \\
CD127-PE-Vio770 & Mouse IgG2a-PE-Vio770 \\
& CD4 (VIT4)-VioBlue & Mouse IgG2a-VioBlue \\
nTregs & CD18-FITC & Mouse IgG1-FITC \\
& CD223-PE & REA Control (S)-PE \\
& CD49b-PE-Vio770 & REA Control (S)-PE-Vio770 \\
& CD152-APC & Mouse IgG2a-APC \\
\hline
\end{tabular}

As shown for the other immune cells, CD4+ T cell frequency decreases after the first session of H-SRT (T1), from $31 \%$ to $17 \%$. Then, it increases again from T2 to T4, to come back to the steady state. When the disease is progressing (T5) CD4+ population drop to $25 \%$. Immediately after Nivolumab injection, the frequency increases again, (35\%) and gradually decreases until T8 (Figure 2A). A similar pattern is observed for CD8+ cells (Figure 2B) and CD11c+ cells (dendritic cells and B cells) (Figure 2C). For CD14+ cells (monocytes), the variation is different during radiotherapy with a decrease at $\mathrm{T} 1$, then an increase at $\mathrm{T} 2$ and a decrease at $\mathrm{T} 3$. However, after $\mathrm{T} 5$ and Nivolumab injection, the evolution of the profile is the same (Figure 2D). For CD355+ cells (NK cells), the only difference observed with CD4+ cells is a decrease at T6, with a more delayed increase, at T7 after Nivolumab injection (Figure 2F).

Dendritic cells subset (CD3-CD11c+CD14low/-) also decreases at T1, increases again at T2 and stabilizes at T3 $(28.1 \%)$. There is a noticeable decrease at T4 and a re-increase at T5 (Figure 3B). After immunotherapy treatment, their frequency decreases immediately to T6 then increases to T7 and stabilizes at T8 (Figure 3A,B).

We also observed a decrease of the B lymphocyte fraction (CD3-CD11c+CD19+), at T1 and then an increase at $\mathrm{T} 2$ and a decrease at $\mathrm{T} 3$. Their proportion therefore varies during radiotherapy. At T4, the rate increases and decreases again at T5 (Figure 3D). After the anti-PD1 introduction, there is a significant increase in the level of lymphocyte B to T6 which remains at T7. Their proportion decreases again to $\mathrm{T} 8$ (Figure 3C,D). 
CD4

A

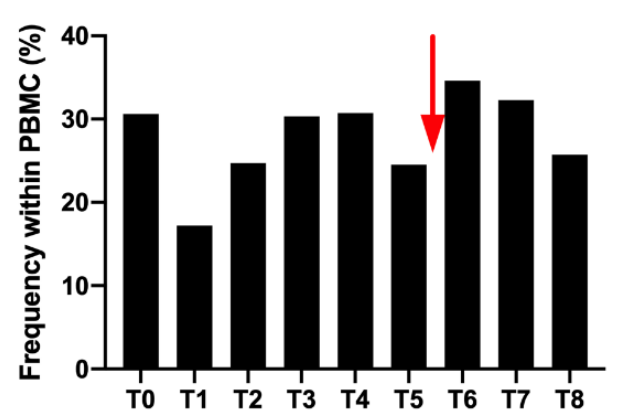

C

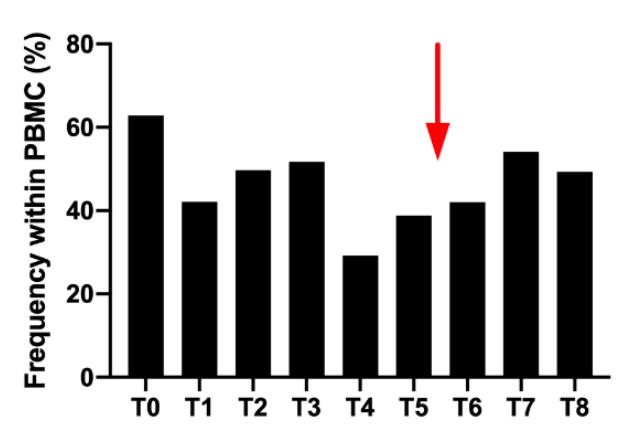

E

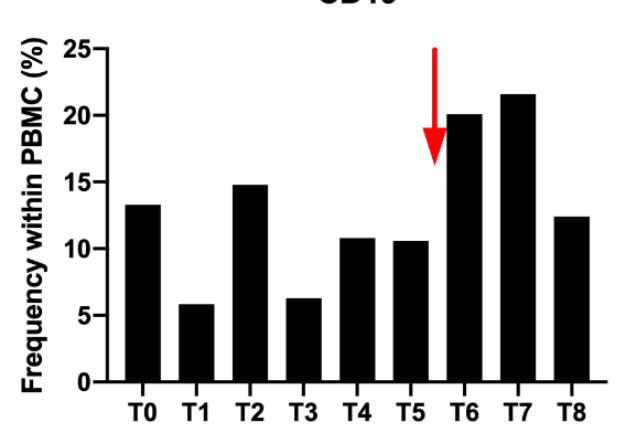

B

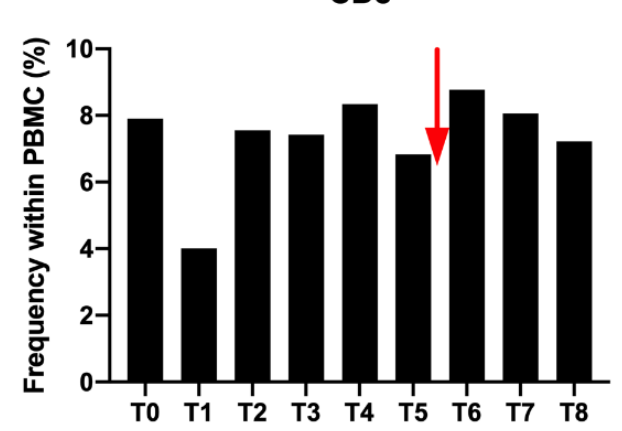

CD14

D

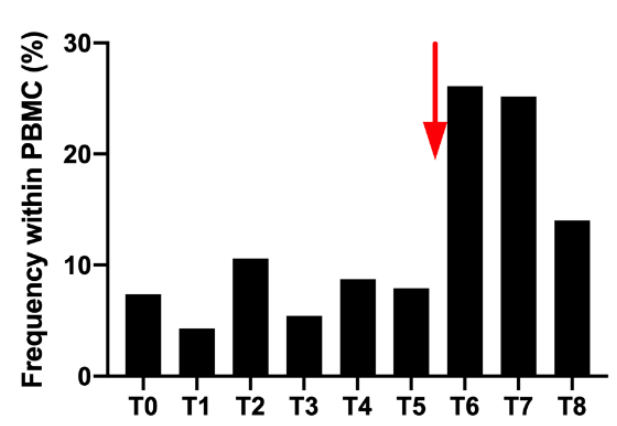

CD335

$\mathbf{F}$

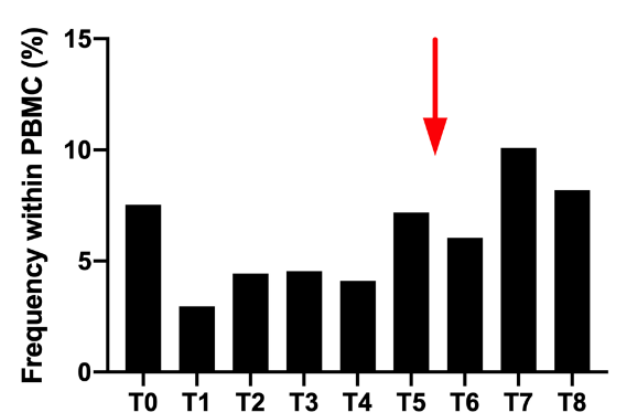

Figure 2. Expression of CD4+ (A), CD8+ (B), CD11C+ (C), CD14+ (D), CD19+ (E) and CD335+ (F) markers among the patient's peripheral blood mononuclear cells. All these results were obtained from flow cytometry assay and expression percentage. The red arrow corresponds to the single injection of Nivolumab. T0 to T8 correspond to all the study time points as described above from before the radiotherapy to 1 year after the last session. 
A.

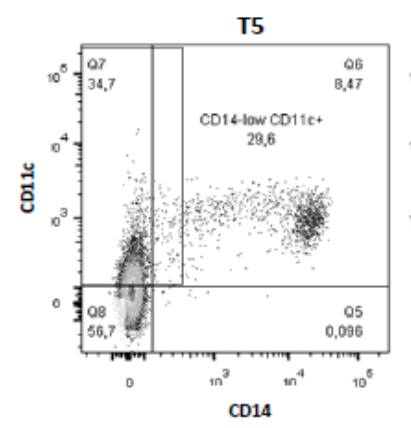

T7

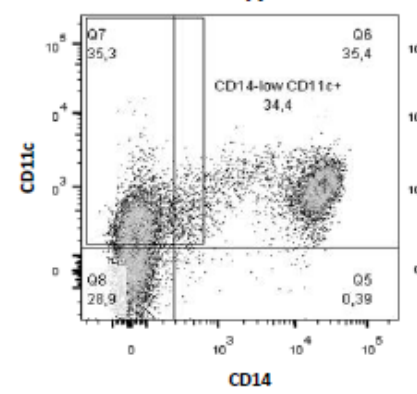

$\mathrm{CD} 14$

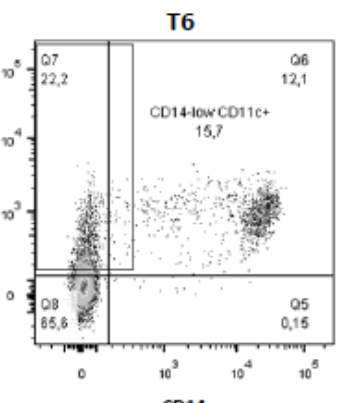

T8

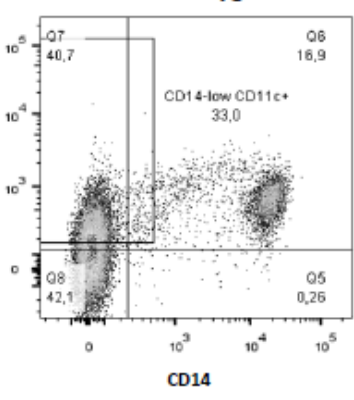

CD14

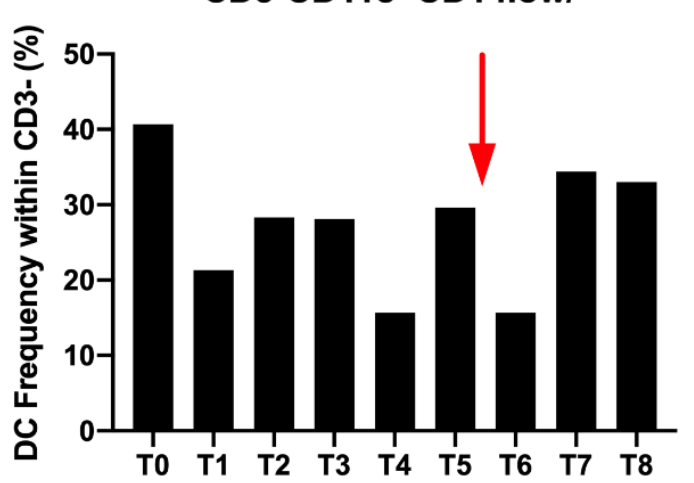

C.
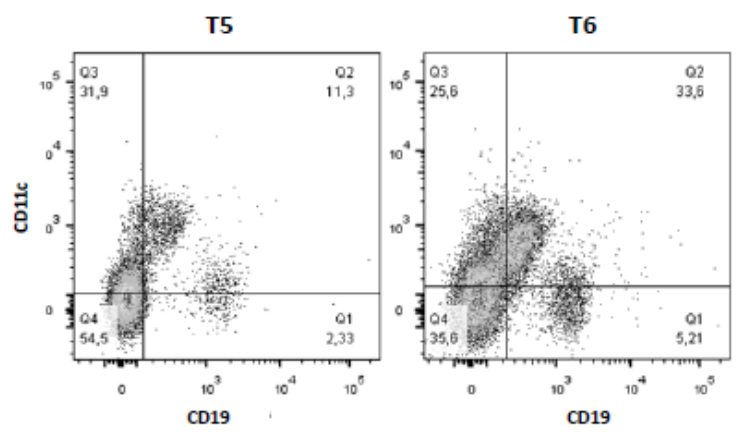

T7

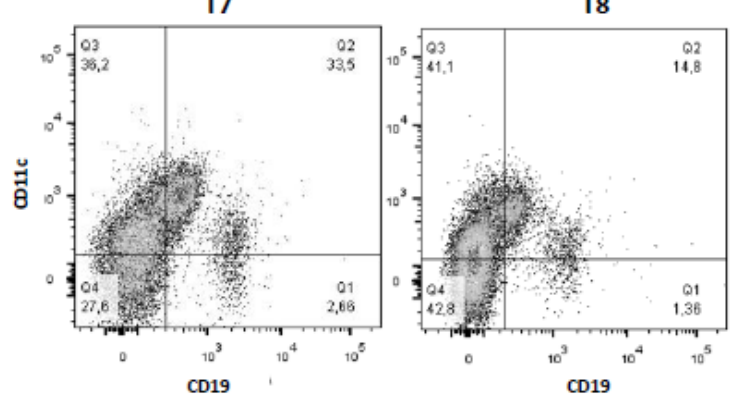

D. CD3-CD11C+CD19+

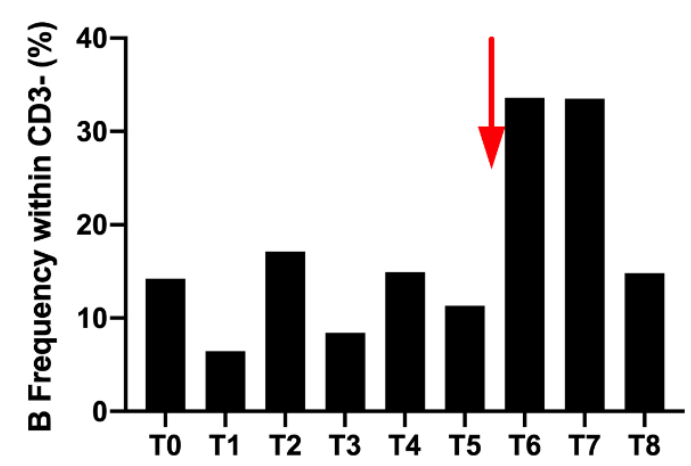

Figure 3. Gating strategy for frequency of CD11c+CD14low/- dendritic cells $(\mathbf{A})$ and CD11c+CD19+ B lymphocytes (C) before (T5) and after (T6-8) Nivolumab injection (red arrow). Frequency of CD11c+CD14low/- dendritic cells (B) and CD11c+CD19+ B lymphocytes (D) in CD3-cells during all the follow up.

Regarding the activation status of the CD4+ T cells during radiotherapy, slight variations in activation markers are observed, except for HLA-DR which increases from T0 to T3 (Figure 4). Very interestingly, after Nivolumab injection, a clear increase in CD25, CD30, CD69 and CTLA-4 activating markers was observed (Figure 4). An increase is also found for CCR7 but less importantly. Conversely, the HLA-DR marker decreases from T5 to T8. 
$[\mathrm{CD} 4+/ \mathrm{CCR} 7+]$

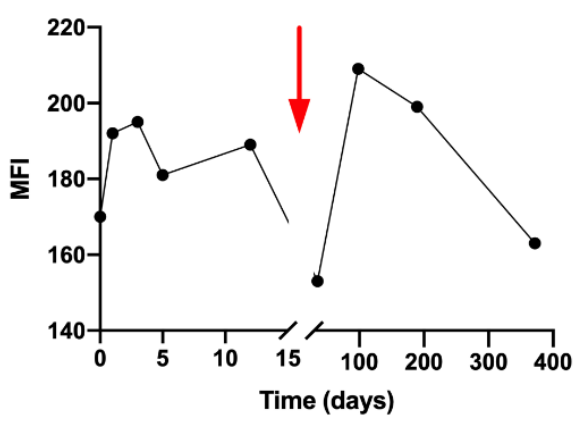

$[\mathrm{CD} 4+/ \mathrm{CD} 30+]$

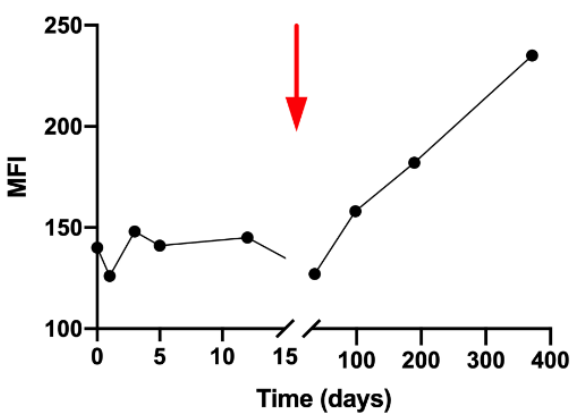

[CD4+/CTLA4+]

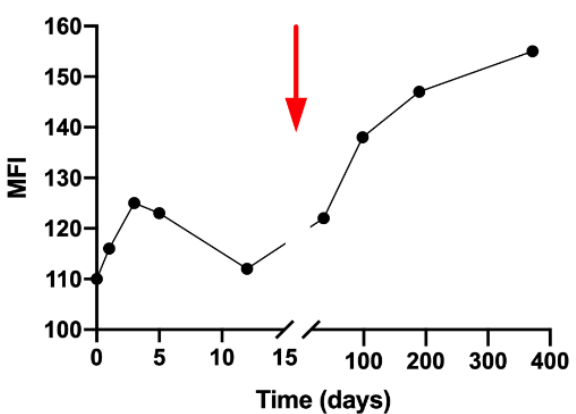

$[\mathrm{CD} 4+/ \mathrm{CD} 25+]$

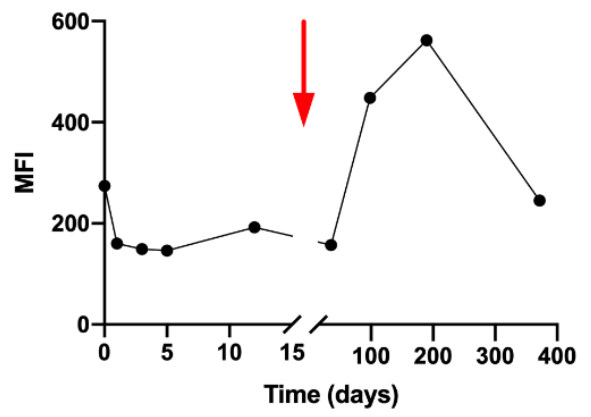

[CD4+/CD69+]

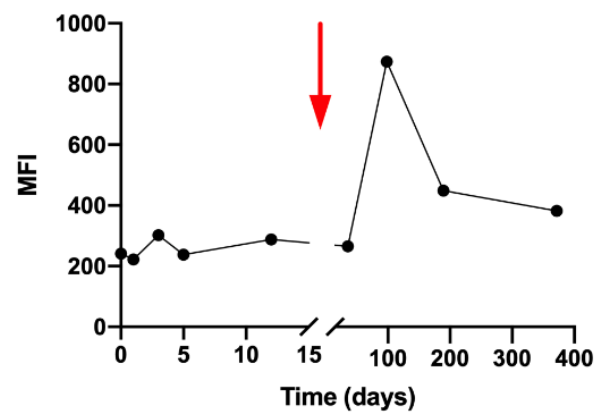

[CD4+/HLADR+]

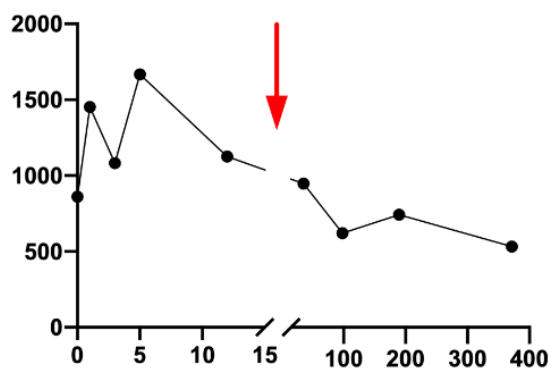

Figure 4. Analysis of CD4+ T lymphocytes activation state by flow cytometry for several activation markers: CCR7, CD25, CD30, CD69, CTLA4, HLADR. Results are presented over time (days) and expressed in median of fluorescence (MFI). The red arrow points the Nivolumab injection timepoint.

Regarding CD8+ T cells activation, during $\mathrm{T} 0$ to $\mathrm{T} 3$, small variations were observed for the CCR7, CD25, CD30, CTL4 and HLA-DR markers (Figure 5). Compared with CD4+ LT, the observed changes are greater after Nivolumab injection after T5 with an increase in all markers: CCR7, CD25, CD30, CD69 and HLA-DR. It can be noted that the variations of the CCR7 marker are similar after the beginning of the radiotherapy and the Nivolumab treatment. 
$[\mathrm{CD} 8+/ \mathrm{CCR} 7+]$

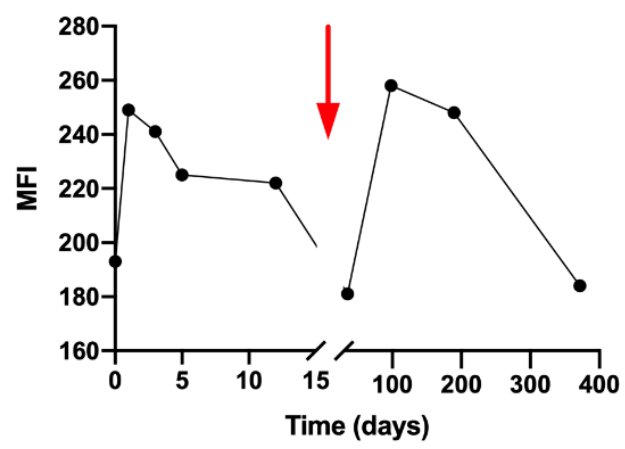

$[\mathrm{CD} 8+/ \mathrm{CD} 30+]$

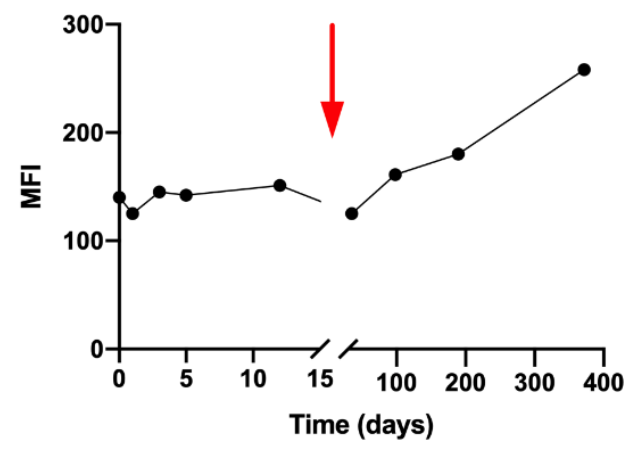

[CD8+/CTLA4+]

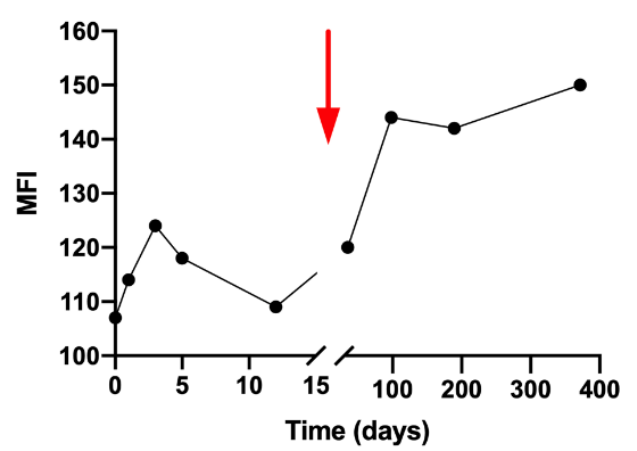

[CD8+/CD25+]

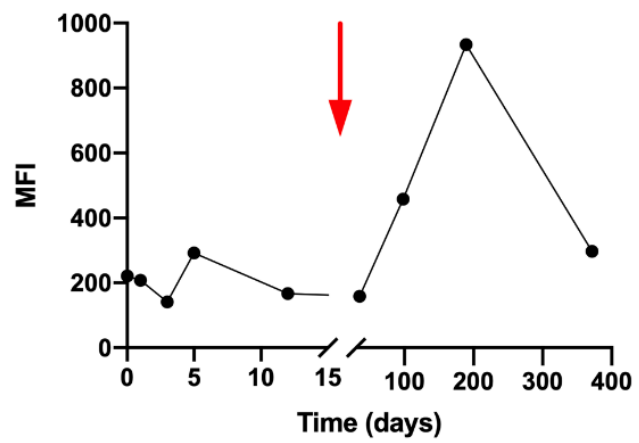

[CD8+/CD69+]

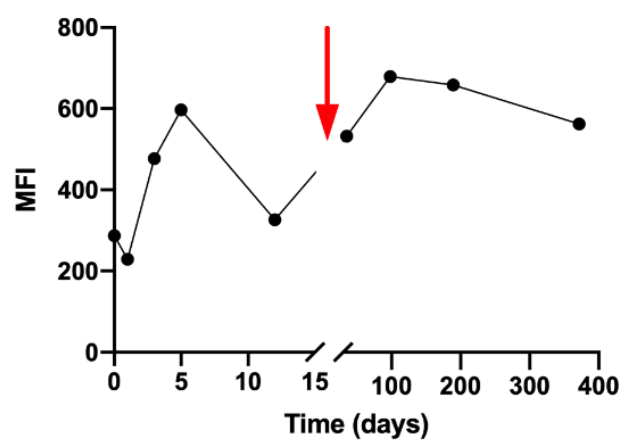

[CD8+/HLADR+]

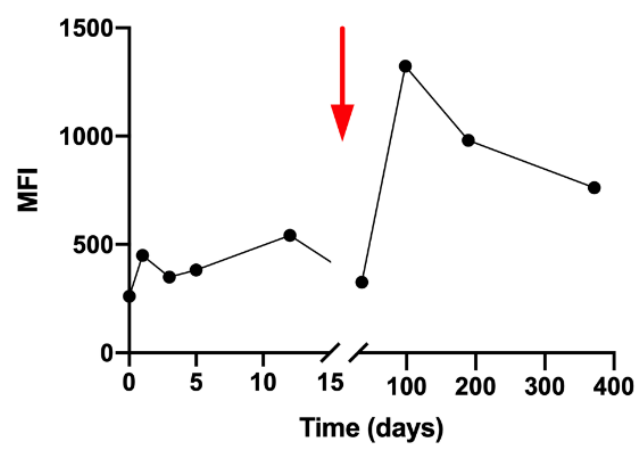

Figure 5. Analysis of CD8+ T lymphocytes activation state by flow cytometry for several activation markers: CCR7, CD25, CD30, CD69, CTLA4, HLADR. Results are presented over time (days) and expressed in median of fluorescence (MFI). The red arrow points the Nivolumab injection timepoint, 90 days after the beginning of the follow up.

The proportion of CD4+CD25highCD127-/low nTregs in CD4 T cells is very low (0.7\%) before treatment (T0). There is an increase to $2.91 \%$ at $\mathrm{T} 1$ and then a decrease from $\mathrm{T} 2$ to $\mathrm{T} 3$. Their proportion remains low during radiotherapy. Then it increases from $\mathrm{T} 4$ to $\mathrm{T} 5$ at $3.15 \%$ and $4.06 \%$, respectively. 
After Nivolumab injection, nTregs collapse at $0.23 \%$. A very large increase at $\mathrm{T} 8$ at $6.68 \%$ at the end of follow-up is observed (Figure 6A).
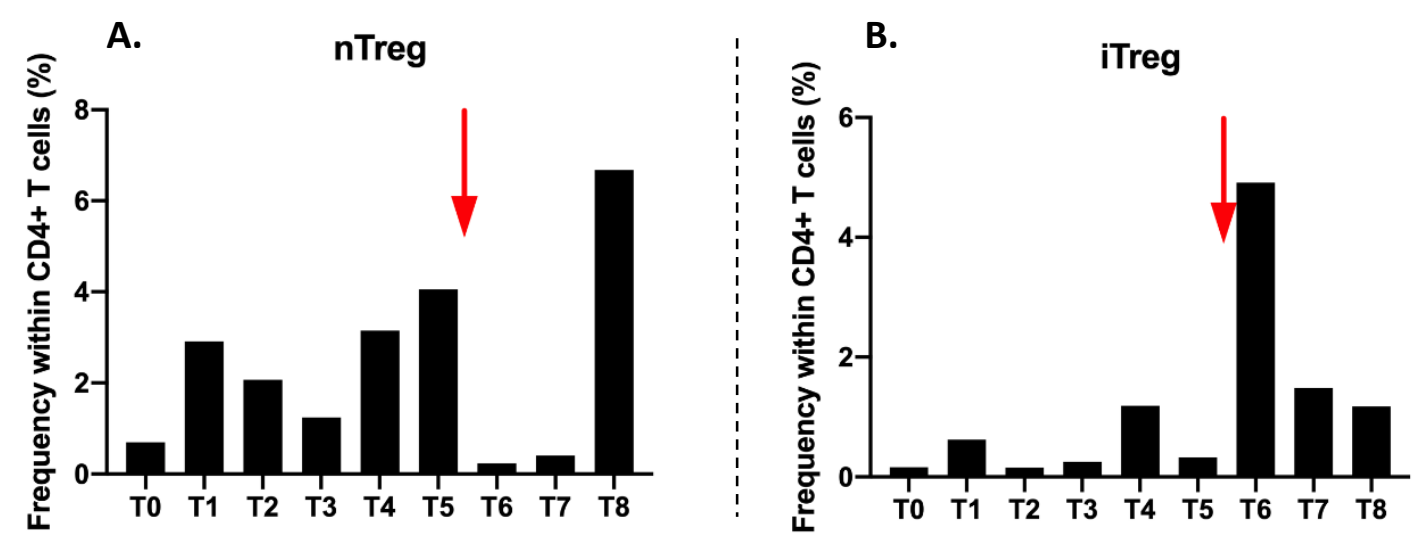

Figure 6. Frequency (in percentage) of CD4+CD25+/highCD127-/low nTreg (A) and frequency of CD4+CD18+CD49b+iTreg lymphocytes in CD4+ cells (B) during all timepoints of the follow up. The red arrow represent the time of the single Nivolumab injection after T5.

The proportion of induced Treg (iTregs) is very low at $0.16 \%$ at T0. It varies during radiotherapy from $\mathrm{T} 0$ to $\mathrm{T} 3$, but in values that remain very low. The major change is observed at $\mathrm{T} 6$ with an increase to $4.91 \%$ just after the injection of Nivolumab. Then the proportions decrease gradually until $\mathrm{T} 8$ to reach $1.18 \%$ (Figure $6 \mathrm{~B}$ ).

\subsection{Activating Cytokine Production after Combination of High Dose Hypofractionated Radiotherapy with Anti-PD1 Single Dose Immunotherapy}

Patient's sera were collected after coagulation in dry tubes (Greiner Bio One Vacuette ${ }^{\circledR}$, Les Ulis, France). Sera were stored at $-80{ }^{\circ} \mathrm{C}$ until the entire sera collection was complete. Enzyme-Linked ImmunoSorbent Assay (ELISA) was used to detect and quantify sera for 8 cytokines (IL10, IL12, IFN- $\gamma$, TGF $\beta$, IL4, IL6, IL2 and TNF $\alpha$ ) (BD Pharmingen, Franklin Lakes, NJ, USA) following manufacturer's recommendations. Readings were made at $492 \mathrm{~nm}$ using the Multiskan spectrometer (Multiskan EX, ThermoLabsystems, France) powered by Ascent Software (ThermoFisher Scientific ${ }^{\mathrm{TM}}$, Walthman, MA, USA).

Regarding Th1 cytokines, IL-2 concentrations are higher than $700 \mathrm{pg} / \mathrm{mL}$ at T0. Variation are observed during radiotherapy. A spontaneous decrease is observed at T5, which increases after Nivolumab injection and the concentration increases again at T8 (Figure 7A). Furthermore, for IFN $\gamma$, a high $\mathrm{T} 0$ concentration of $547 \mu \mathrm{g} / \mathrm{mL}$ is also observed. Then, there is a profile similar to IL2 with a slight decrease followed by an increase during radiotherapy (Figure 7B). At T5, the concentration decreases. The concentration increases again to T8. No value for TNF $\alpha$ was detected in ELISA.

Il-12 is a cytokine predominantly secreted by activated dendritic cells. Its concentration is raised to $404 \mathrm{pg} / \mathrm{mL}$ at the beginning of the treatment and remains stable during the radiotherapy treatment. On the other hand, there is a decrease after the injection of Nivolumab until T7 $(158 \mu \mathrm{g} / \mathrm{mL})$ and a final slight increase at T8 (Figure 7C).

Regarding Th2 cytokines only a low IL-4 concentration is detected (Figure 7D). Nevertheless, by looking at the overall evolution, Th2 cytokines are similarly observed and the concentrations decrease after Nivolumab injection. The concentration finally increases again to T8. However, in the immediate aftermath of T4 and T6 radiotherapy, there was no detectable IL-6 (Figure 7E). Values increase again at $\mathrm{T} 7$ and T8 to find respective values at $1760 \mu \mathrm{g} / \mathrm{mL}$ and $2240 \mu \mathrm{g} / \mathrm{mL}$, higher than T0 (Figure 7E). 

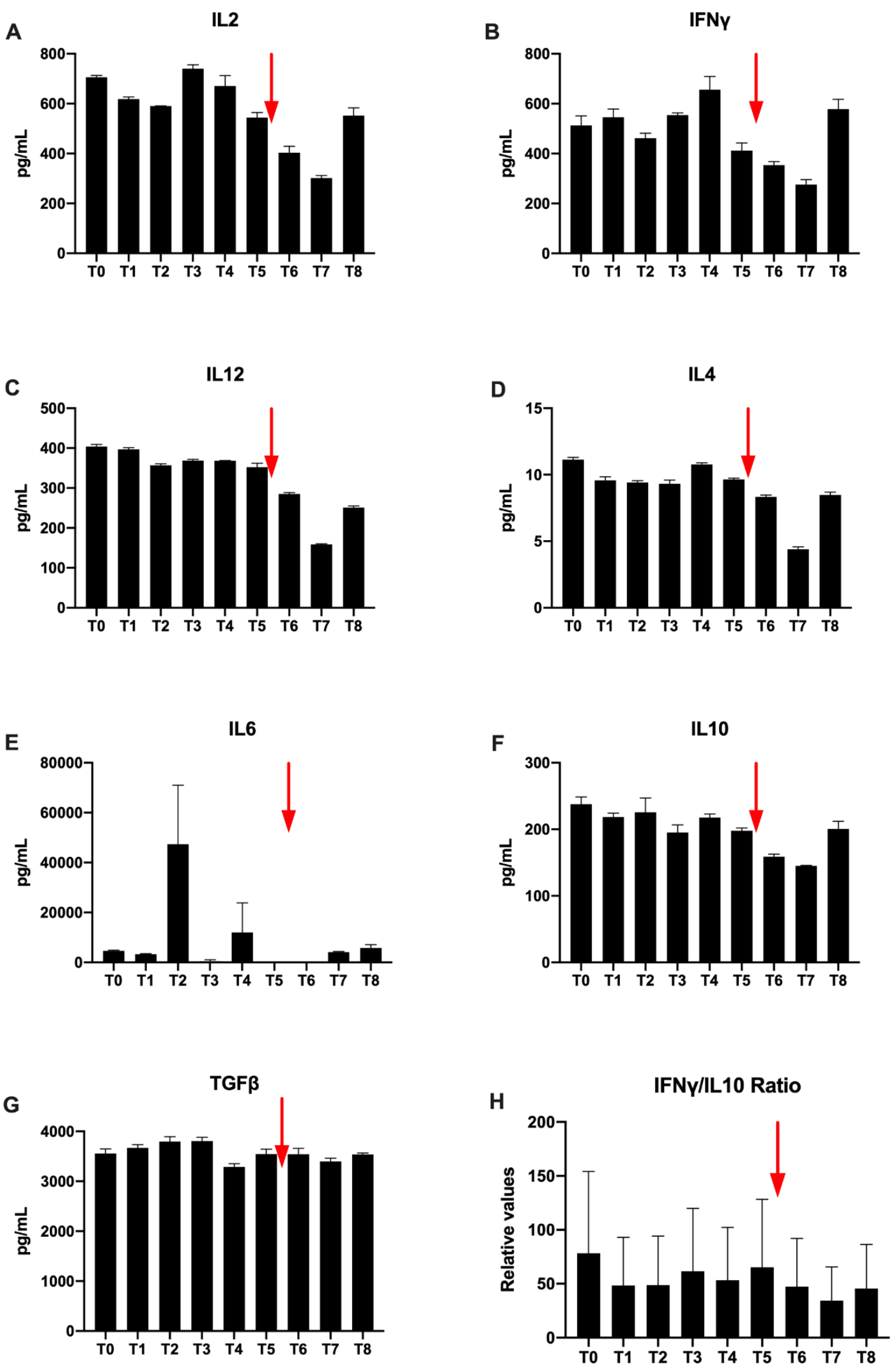

Figure 7. Secretion of IL2 (A), IFN $\gamma$ (B) and IL12 (C) Th1 cytokines, IL4 (D) and IL6 (E) Th2 cytokines and IL10 (F) and TGF $\beta$ (G) immunosuppressive cytokines in patient's sera. represented in $\mathrm{pg} / \mathrm{mL}$. Representation of IFN $\gamma /$ IL10 ratio (relative values) $(\mathbf{H})$. The time of the Nivolumab single dose injection is represented by the red arrow. 
Regarding, immunosuppressive cytokines, the concentrations vary faintly during radiation therapy (Figure 7F,G). Before H-SRT TGF $\beta$, concentration is very high $(3550 \mu \mathrm{g} / \mathrm{mL})$ (Figure 7G). It increases during radiotherapy. Then, a concentration decrease is observed. Concentrations vary only slightly between T5 and T6. The last TGF $\beta$ concentration is equivalent compared to T0 (Figure 7G). For IL10, a spontaneous decrease is observed at T5 at $148 \mathrm{pg} / \mathrm{mL}$, which increases after Nivolumab injection from T6 to T7 (Figure 7F).

Finally, the IFN $\gamma / \mathrm{IL}-10$ ratio, used in immunology to compare anti-tumor versus immunosuppressive cytokines, is in favor of the anti-tumor cytokine (IFN $\gamma$ ). This ratio is not changed during radiotherapy. It increases gradually after the Nivolumab injection at the end of follow-up; from $\mathrm{T} 7$ to reach a final value greater than 5 to $\mathrm{T} 8$ (Figure $7 \mathrm{H}$ ).

2.5. Modulation of the Effect of the Patient's Exosomes on Immune Cell Proliferation After Combination of High Dose Hypofractionated Radiotherapy with Anti-PD1 Single Dose Immunotherapy

Exosomes were isolated from plasma after several centrifugation and ultracentrifugation. They are selected using a sucrose cushion as described in a previous publication [18]. After isolation exosomes were stocked at $-80^{\circ} \mathrm{C}$. Exosomes were then dosed using the colorimetric Bradford method (BIORAD Laboratories, Hercules, CA, USA). For functional assay PBMC were activated by $1 \mu \mathrm{g} / \mathrm{mL}$ of Phytohemagglutinin (PHA) (Sigma, St Louis, MO, USA) before to be co-incubated with $3.33 \mu \mathrm{g} / \mathrm{mL}$ of the patient's exosomes.

After $72 \mathrm{~h}$ of total incubation with exosomes, PBMC proliferation was analyzed after $18 \mathrm{~h}$ of incubation with [methyl-3H]-thymidine $(1 \mu \mathrm{Ci} /$ well) (PerkinElmer, Waltham, MA, USA). The incorporation was evaluated using a 1450 Trilux luminometer (Perkin Elmer, Walthman, MA, USA) after the filtration of plates in a glass fiber filter (Perkin Elmer, Walthman, MA, USA) and the addition of liquid scintillation (Beckman, Brea, CA, USA). Results are the mean of triplicates and are expressed in count per minute (CPM).

After HRT, we first observed at T1, T2 and T3 a decrease of the PBMC proliferation when they were co-cultured with exosomes isolated from the melanoma patient, followed by an increase of this proliferation since T3 to T6. However, just after Nivolumab injection, we observed a shift of the exosome impact on immune cells (Figure 8). Indeed, we clearly observed after the Nivolumab treatment a decrease of the immune activating potential of the newly produced exosomes.

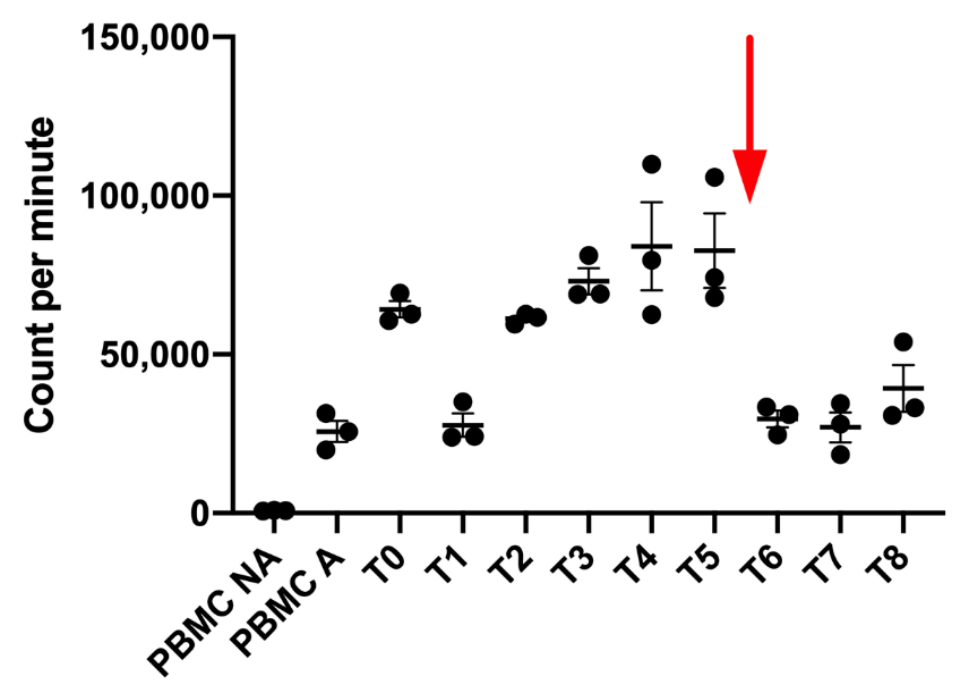

Figure 8. Patient's Exosomes effect on regulation of activated PBMC (PHA $1 \mu \mathrm{g} / \mathrm{mL})$. Activated PBMC only (PBMC A) use as positive control and non-activated PBMC (PBMC NA) as negative control. The red arrow represent time of the single dose injection of Nivolumab. 


\section{Discussion}

Immune checkpoint inhibitors (ICIs) have revolutionized cancer treatment. Nivolumab (anti-PD1 monoclonal antibody) has been shown to be effective in metastatic melanoma treatment. However, despite the increase in overall survival in $33-40 \%$ of melanoma patients, anti-PD1 treatment did not show efficacy in the majority of treated patients $[19,20]$. Indeed, the effectiveness of these treatments varies greatly depending on the individual and the type of cancer. This raises the question of therapeutic combinations, particularly with radiotherapy, which would be rationally able to modify the immunological tumor characteristics [8]. Early studies have shown encouraging results [17]. Thus, in recent years, extraordinary efforts have been devoted to identifying predictive biomarkers' response, some of which are already used in clinical settings for decision-making as BRAF and KIT mutation status for melanoma [21]. Circulating biomarkers are, therefore, of great interest because of their inexpensiveness, accessibility and non-invasive nature.

In this context, the aim of this study was to evaluate the immune response regulation during a high-dose hypo fractionated radiotherapy followed by a Nivolumab therapy in a patient with metastatic melanoma and to correlate biological data and clinical response. The goal was to highlight circulating immune biomarkers of response to treatments.

Overall, results showed lymphocyte activation after radiotherapy, followed by a decrease in activation during cancer relapse. However, after the introduction of a single injection the immune check point inhibitor (anti-PD1), patient immune status was increased in favor of an immune activation. Furthermore, the patient experienced adverse events after each treatment received indicating a systemic response. Vitiligo is an adverse effect correlated with a good response to immunotherapy [22]. Up to now, the patient presents a complete clinical response.

In this study, before treatment (T0), the patient has a rather favorable immune environment. Indeed, high immune subpopulation proportions within PBMCs are observed. nTregs and iTregs proportions are very low. Anti-tumor cytokines IFNy and IL-2 also have high values and the IFNy/IL10 ratio is greater than 2 .

Nevertheless, after radiotherapy (T1) the different immune sub-population analysis reveals an immunosuppression. This immunosuppression is reversible because of immune sub-populations restoration until T4. For this patient, immune populations fall at T5. Interestingly, this status is clinically correlated with patient stability at $\mathrm{T} 4$ and patient clinical progress at $\mathrm{T} 5$.

There is also effective immune response establishment. Indeed, dendritic cells proportion (CD3-CD11c+CD14low/-) increases during H-SRT after T1. A transcriptomic analysis also showed an increase in DC maturation markers gene expression (CD80, CD83, CD86), which are witnesses of a previous antigen capture by DCs and their future maturation status (data not shown). LT CD4 and LT CD8 proportions increase also, even though there is small variation in activation markers. However, this immune response boost after radiotherapy seems transient, as a decrease in all these markers appears; it corresponds to the patient's cancer progression.

Radiation therapy is, therefore, responsible of an early activation of the immune system. This immune activation seems to correlate to the clinic regarding patient initial symptomatology (chills, vitiligo) as well as good local control. However, this activation is transient. Indeed, at T5 an unfavorable profile is observed with a decrease in most cell populations. After Nivolumab introduction between $\mathrm{T} 5$ and $\mathrm{T} 6$, there was an increase in all immune subpopulations, except for NK cells and DC, and more importantly for CD4+, CD14+ and CD19+. Immune system activation is supported by the analysis of activation markers, which increase after $\mathrm{T} 5$ for all studied ones.

iTregs increase at T6 could be explained by the tumor antigen release after anti-PD1 treatment. In addition, the need to restore cellular homeostasis and patient immune functions lead to nTregs increase in T8. However, during treatment, if many antigens are released then DCs will mature later and/or migrate to the nearest draining lymph nodes or mature during their course to secondarily activate the adaptive response. 
Unexpectedly, a significant increase in B-cells after immunotherapy is observed. Nevertheless, it has been reported that PD1 is expressed at the lymphocyte level and it has been shown in a mouse model that the PD-1 antigen expression is tightly regulated and induced by signal transduction across the antigen receptor [23]. The possibility that the PD-1 antigen may play a role in clonal selection of B-cells is not excluded. Accordingly, this increase could also be related to the Th2 immune response observed after the introduction of Nivolumab with IL-4 and IL-6 cytokines.

Very interestingly, we showed for the first time that a patient's exosomes secreted after H-SRT are in favor of an immune activation with an increase of the proliferation of immune cells while those secreted after a Nivolumab single dose did not activate or inhibit immune cell proliferation. This observation is quite interesting as it is the opposite of clinical observation. It seems to show that the good response after a Nivolumab injection is probably not mediated by exosomes.

Immediately after immunotherapy introduction, an increase in T cell lymphocytes, CD14+ monocytes, LB and iTregs is observed. Nivolumab may also induce tolerogenic DCs. Nevertheless, additional markers, particularly of DC differentiation and maturation, have to be explored to validate this hypothesis. LB increase has not been described under immunotherapy as LBs have never been described as potential biomarkers of anti-PD1 immunotherapy efficacy. Nevertheless, the expression of PD1 by the LB could be an interesting element to explore.

\section{Conclusions}

In conclusion, although we noted that this case study did not allow for the identification of an early biomarker of response to treatment, all results obtained in this exceptional case open an interesting therapeutic perspective and raise the question of the necessity of combining therapies to optimize the response to treatments. Indeed, it seems that the combination of H-SRT with anti-PD1 immunotherapy may favor an activation of the immune response which could result in a better clinical response. The results of this pilot study are very encouraging, but it should be validated on a larger cohort. The advent of immunotherapy has brought about a paradigm shift in the advanced cancer treatment landscape. These novel therapeutics may result in unconventional response patterns and, therefore, special strategies are needed for an accurate assessment of tumor responses and clinical outcomes. Development of robust biomarkers to identify responder patients and monitor immunotherapy response is a key step for further progress in this area and the development of precision immuno-oncology approaches. The circulating biomarkers analyzed in our study have the advantage of being easily evaluable in common tests used in clinics and are also suitable for serial sampling during treatment. However, given the complexity of tumor-host interactions and the dynamic nature of most immunologic biomarkers, it is not surprising that each of them has shown limited value when used individually. Efforts are needed to develop integrative models that consider multiple tumor and host parameters and characteristics, such as the "cancer immunogram" [9]

In this precision medicine era, predictive biomarkers are becoming critical to treatment decisions, but this component is not sufficiently integrated in most clinical studies. Future studies involving the systematic and prospective collection of matched blood and tumor samples are needed to confirm these signatures in larger and multicenter cohorts. A predictive signature could then be used directly in clinical practice to stratify patients before starting immunotherapy.

Funding: This research was funded by SANTHELYS (EARLYBIOSANTHE), Canceropôle Nord-Ouest (EARLYBIOCNO), ANRT (ANRTCM), Immune insight (EARLYBIOIST).

Acknowledgments: We would like to acknowledge Santhelys, Siric ONCOLILLE and Cancéropôle Nord-Ouest for their financial Support. We would also would like to thank the ANRT and Immune insighT for the PhD Grant of Clara Milhem (First author).

Conflicts of Interest: The authors declare no conflict of interest. 


\section{References}

1. Strojan, P. Role of radiotherapy in melanoma management. Radiol. Oncol. 2010, 44, 1-12. [CrossRef] [PubMed]

2. Hanahan, D.; Weinberg, R.A. Hallmarks of cancer: The next generation. Cell 2011, 144, 646-674. [CrossRef] [PubMed]

3. Dunn, G.P.; Bruce, A.T.; Ikeda, H.; Old, L.J.; Schreiber, R.D. Cancer immunoediting: From immunosurveillance to tumor escape. Nat. Immunol. 2002, 3, 991-998. [CrossRef] [PubMed]

4. Schreiber, R.D.; Old, L.J.; Smyth, M.J. Cancer Immunoediting: Integrating Immunity's Roles in Cancer Suppression and Promotion. Science 2011, 331, 1565-1570. [CrossRef] [PubMed]

5. Supiot, S.; Clément-Colmou, K.; Paris, F.; Corre, I.; Chiavassa, S.; Delpon, G. Which rules apply to hypofractionated radiotherapy? Cancer Radiother. 2015, 19, 421-425. [CrossRef]

6. Brown, J.M.; Koong, A.C. High-dose single-fraction radiotherapy: Exploiting a new biology? Int. J. Radiat. Oncol. Biol. Phys. 2008, 71, 324-325. [CrossRef]

7. Mole, R.H. Whole body irradiation; radiobiology or medicine? Br. J. Radiol. 1953, 26, 234-241. [CrossRef]

8. De Meerleer, G.; Khoo, V.; Escudier, B.; Joniau, S.; Bossi, A.; Ost, P.; Briganti, A.; Fonteyne, V.; Van Vulpen, M.; Lumen, N.; et al. Radiotherapy for renal-cell carcinoma. Lancet Oncol. 2014, 15, e170-e177. [CrossRef]

9. Blank, C.; Gajewski, T.F.; Mackensen, A. Interaction of PD-L1 on tumor cells with PD-1 on tumor-specific $\mathrm{T}$ cells as a mechanism of immune evasion: Implications for tumor immunotherapy. Cancer Immunol. Immunother. 2005, 54, 307-314. [CrossRef]

10. Parekh, V.V.; Lalani, S.; Kim, S.; Halder, K.; Azuma, M.; Yagita, H.; Kumar, V.; Wu, L.; Kaer, L.V. PD-1/PD-L blockade prevents anergy induction and enhances the anti-tumor activities of glycolipid-activated invariant NKT cells. J. Immunol. 2009, 182, 2816-2826. [CrossRef]

11. Talay, O.; Shen, C.-H.; Chen, L.; Chen, J. B7-H1 (PD-L1) on T cells is required for T-cell-mediated conditioning of dendritic cell maturation. Proc. Natl. Acad. Sci. USA 2009, 106, 2741-2746. [CrossRef] [PubMed]

12. Ngwa, W.; Irabor, Q.C.; Schoenfeld, J.D.; Hesser, J.; Demaria, S.; Formenti, S.C. Using immunotherapy to boost the abscopal effect. Nat. Rev. Cancer 2018, 18, 313-322. [CrossRef] [PubMed]

13. Formenti, S.C.; Demaria, S. Combining radiotherapy and cancer immunotherapy: A paradigm shift. J. Natl. Cancer Inst. 2013, 105, 256-265. [CrossRef]

14. Vacchelli, E.; Enot, D.P.; Pietrocola, F.; Zitvogel, L.; Kroemer, G. Impact of Pattern Recognition Receptors on the Prognosis of Breast Cancer Patients Undergoing Adjuvant Chemotherapy. Cancer Res. 2013, 76, 3122-3126. [CrossRef]

15. Sharabi, A.B.; Nirschl, C.J.; Kochel, C.M.; Nirschl, T.R.; Francica, B.J.; Velarde, E.; Deweese, T.L.; Drake, C.G. Stereotactic Radiation Therapy Augments Antigen-Specific PD-1-Mediated Antitumor Immune Responses via Cross-Presentation of Tumor Antigen. Cancer Immunol. Res. 2015, 3, 345-355. [CrossRef] [PubMed]

16. Park, S.S.; Dong, H.; Liu, X.; Harrington, S.M.; Krco, C.J.; Grams, M.P.; Mansfield, A.S.; Furutani, K.M.; Olivier, K.R.; Kwon, E.D. PD-1 Restrains Radiotherapy-Induced Abscopal Effect. Cancer Immunol. Res. 2015, 3, 610-619. [CrossRef]

17. Liniker, E.; Menzies, A.M.; Kong, B.Y.; Cooper, A.; Ramanujam, S.; LO, S.; Kefford, R.F.; Fogarty, G.B.; Guminski, A.; Wang, T.W.; et al. Activity and safety of radiotherapy with anti-PD-1 drug therapy in patients with metastatic melanoma. Oncoimmunology 2016, 5, e1214788. [CrossRef]

18. Mrizak, D.; Martin, N.; Barjon, C.; Jimenez-Pailhes, A.-S.; Mustapha, R.; Niki, T.; Guigay, J.; Pancré, V.; de Launoit, Y.; Busson, P.; et al. Effect of Nasopharyngeal Carcinoma-Derived Exosomes on Human Regulatory T Cells. J. Natl. Cancer Inst. 2014, 107, 363.

19. Robert, C.; Schachter, J.; Long, G.V.; Arance, A.; Grob, J.J.; Mortier, L.; Daud, A.; Carlino, M.S.; McNeil, C.; Lotem, M.; et al. Pembrolizumab versus Ipilimumab in Advanced Melanoma. N. Engl. J. Med. 2015, 372, 2521-2532. [CrossRef]

20. Ribas, A.; Hamid, O.; Daud, A.; Hodi, F.S.; Wolchok, J.D.; Kefford, R.; Joshua, A.M.; Patnaik, A.; Hwu, W.-J.; Weber, J.S.; et al. Association of Pembrolizumab with Tumor Response and Survival among Patients with Advanced Melanoma. JAMA 2016, 315, 1600-1609. [CrossRef] [PubMed]

21. Nebhan, C.A.; Johnson, D.B. Predictive biomarkers of response to immune checkpoint inhibitors in melanoma. Expert Rev. Anticancer Ther. 2020, 20, 137-145. [CrossRef] [PubMed] 
22. Nakamura, Y.; Tanaka, R.; Asami, Y.; Teramoto, Y.; Imamura, T.; Sato, S.; Maruyama, H.; Fujisawa, Y.; Matsuya, T.; Fujimoto, M.; et al. Correlation between vitiligo occurrence and clinical benefit in advanced melanoma patients treated with Nivolumab: A multi-institutional retrospective study. J. Dermatol. 2017, 44, 117-122. [CrossRef] [PubMed]

23. Agata, Y.; Kawasaki, A.; Nishimura, H.; Ishida, Y.; Tsubata, T.; Yagita, H.; Honjo, T. Expression of the PD-1 antigen on the surface of stimulated mouse $\mathrm{T}$ and B lymphocytes. Int. Immunol. 1996, 8, 765-772. [CrossRef] [PubMed]

(C) 2020 by the authors. Licensee MDPI, Basel, Switzerland. This article is an open access article distributed under the terms and conditions of the Creative Commons Attribution (CC BY) license (http://creativecommons.org/licenses/by/4.0/). 